\title{
Autorenspiegel
}

\section{Christiane Giffhorn}

1949 geboren in Rotenburg/Wümme

1968 Abitur an einem Naturwissenschaftlichen Gymnasium in Celle

1968-1969 Studium generale am Leibnitz-Kolleg in Tübingen

1969 Jura-Studium in Tübingen, seit 1971 in Göttingen

1972 Abbruch des Jurastudiums, verschiedene Gelegenheitsjobs als Kellnerin und Verkäuferin

1973-1974 Arbeit als „Bezugsperson“ in einem Kinderladen

1974/77 Studium der Erziehungswissenschaft in Göttingen

\section{Wolfgang Keckeisen}

1946 geboren in Stuttgart; aufgewachsen in Stuttgart (bis 1963) und Frankfurt

1966/67 Ziviler Ersatzdienst

1967/74 Studium der Erziehungswissenschaft, Soziologie, Psychologie und Rechtswissenschaft in Frankfurt

1973 Diplom in Pädagogik

1974/77 Wiss. Assistent am Pädagogischen Seminar der Universität Göttingen

\section{Klaus Mollenhauer}

1928 geboren in Berlin

1944/48 Luftwaffenhelfer, Gefangenschaft, Abitur

1948/50 Studium an der PH Göttingen

1950/52 Volksschullehrer in Bremen

1952/58 Studium der Pädagogik, Soziologie und Literaturwissenschaft in Hamburg und Göttingen, Promotion

1958/65 Wissenschaftlicher Assistent bzw. Akademischer Rat in Göttingen und Berlin (Freie Universität)

1965/77 Prof. für Pädagogik an der PH Berlin (1965) und an den Universitäten Kiel (1966), Frankfurt (1969) und Göttingen (1972).

\section{Michael Parmentier}

1943

geboren in Frankfurt

1963

Abitur

1963-1968 Studium der Germanistik, Geschichte, Philosophie, Staatsexamen

1969-1978 Studium der Erziehungswissenschaften in Frankfurt, Tutor

1972/77 Wiss. Assistent am Pädagogischen Seminar der Universität Göttingen

(c) Springer Fachmedien Wiesbaden GmbH, ein Teil von Springer Nature 2021

K. Mollenhauer, Pädagogik der ,Kritischen Theorie', Neuere Geschichte

der Pädagogik, https://doi.org/10.1007/978-3-658-23246-7_2 